\title{
Identification of Fingerprint using Discrete Wavelet Packet Transform
}

\author{
Fahima Tabassum \\ Institute of Information \\ Technology \\ Janagirnagar University \\ Savar, Dhaka, Bangladesh.
}

\author{
Md. Imdadul Islam \\ Department of Computer \\ Science and Engineering \\ Jahangirnagar University \\ Savar, Dhaka, Bangladesh
}

\author{
M.R. Amin \\ Department of Electronics and \\ Communications Engineering \\ East West University \\ Dhaka, Bangladesh
}

\begin{abstract}
Objective of this paper is to identify a person taking fingerprint as a biometric parameter using wavelet packet transform. Here both conventional discrete wavelet transform (DWT) and discrete wavelet packet transform (WPT) are used considering special basis function/matrix to extract the coefficients of basis functions those convey the most of the energy of the signal or image. Here top 5\% coefficients are chosen which actually convey the characteristics of an image. The outcome of the paper is to determine the set of energetic coefficients of basis functions which carry the features of an image hence storage required to preserve the template of images will be reduced considerably.
\end{abstract}

\section{Keywords}

Signal space, scaling and shifting parameter, basis function, concentrator vector and filter bank.

\section{INTRODUCTION}

Wavelet is an oscillatory function of finite duration has two important characteristics: the average amplitude is zero and most of the energy is confined within a narrow region. For example a sinusoidal wave modulated by a Gaussian pulse provides a wavelet. Fourier transform breaks up a signal into sine waves of infinite number of frequencies and amplitudes. Wavelet transform breaks up a signal into the shifted and scaled versions of the original (or mother) wavelet. Most of the application of wavelet transform in image processing is found as denoising of image [1-2]. DWT and WPT both are used in image compression for example JPEG 2000 uses discrete wavelet transform for image compression. In [3-4] the authors deal with compression of image using the best tree determined on the basis of Shannon entropy is used in compression of an image. Similar analysis is found in feature extraction of one dimensional signal (Biomedical signal) in [5]. In this paper, the objective is extraction of features of an image rather than compression or denoising of it. Most widely used technique in feature extraction of an image is to truncate the high frequency component of an image based on Gabor filter then prevalent algorithms are applied on the filtered image discussed in [6$10]$.

In this paper, a WPT based approach is proposed for verification of fingerprint in criminal investigation. Several approaches of fingerprint matching are found in recent literatures. One approach is to take the 9th level DWT of the original fingerprint image; where the slopes of the three linear lines are stored in matrix form as a template for further comparison. This method gives about 95\% recognition rate explained in [11]. An image based automatic fingerprint matching approach is proposed in [12] where fingerprint images are matched based on the features extracted in the wavelet domain. Another, hierarchical fingerprint matching system is proposed in [13] that utilized features at three levels: Level 1 (pattern), Level 2 (minutia points) and Level 3 (pores and ridge contours), extracted from high resolution fingerprint scans. Here Gabor filters and wavelet transform are used to automatically extract the Level 3 features and are locally matched using Iterative Closest Point (ICP) algorithm. K. Thaiyalnayaki et al [14] propose a combination of features (standard deviation, kurtosis, and skewness) for multi-scale and multi-directional recognition of fingerprint. Fingerprint identification using Wavelet Transform (WT) and Probabilistic Neural Network (PNN) is proposed in [15] where the feature vector is obtained by performing one-dimensional DWT. Gabor Filter based fingerprint classification using support vector machines (SVM) is proposed in [16]. A one step method using Gabor filters for directly extracting fingerprint features from grey level images for a small scale fingerprint recognition system is introduced by C.J. Lee and S.D. Wang [17]. In [18], an identification system uses a gray level watershed method based on edge detection to find out the ridges present on a particular fingerprint image for comparison. B. Tan et al [19] present a detection method based on noise analysis along the valleys in the ridge-valley structure of fingerprint images. A neural network based method is applied and trained in [20] throughout the fingerprint skeleton to locate various minutiae.

The paper is organized like: section 2 provides the concept DWT and WPT with example, section 3 provides two proposed algorithm to extract the characteristic of a fingerprint, section 4 deals with the result corresponding to the proposal of section 3 and finally section 5 concludes the entire analysis.

\section{SERIES EXPANSION}

This section will provide the concept of DWT and WPT under signal space and the way of implementation of both the techniques using filter bank of FIR filter. Consider a vector $\mathbf{X}$ in vector space $V_{N}$ and the corresponding vector in subspace $V_{N-1}$ becomes $\mathbf{X}_{\mathrm{N}-1}$ (excluding the $N$ th basis function). Here the orthogonal projection of $\mathbf{X}$ (under vector space $V_{N}$ ) is considered on signal space $V_{N-1}$ as $\mathbf{X}_{\mathrm{N}-1}$ and $\mathbf{X}_{\mathbf{N}-1}$ is the closest approximation of $\mathbf{X}_{\mathbf{N}}$. Therefore the error vector, $\mathbf{e}_{\mathrm{N}-1}=\mathbf{X}-\mathbf{X}_{\mathrm{N}-1}$. Now the vector $\mathbf{X}$ can be expressed like: $\mathbf{X}=\mathbf{e}_{N-1}+\mathbf{e}_{N-2}+\cdots \quad \ldots \quad \ldots+\mathbf{e}_{1}+\mathbf{X}_{1}$. If $W_{l}, W_{2}, W_{3}, \ldots \ldots W_{N-l}$ are the one dimensional vector space and orthogonal projection of $\mathbf{X}_{\mathbf{K}}$ on $W_{K}$ is $e_{k}$ then the vector space is expressed as:

$V_{N}=W_{N-1} \oplus W_{N-2} \oplus \cdots \cdots \cdots \cdots \oplus W_{1} \oplus V_{1}$

(1) 
In wavelet transform two basis functions $\varphi_{u, k}(x)$ and $\psi_{j, k}(t)$ are used, called scaling and wavelet function corresponding to subspace $V_{u}$ and $W_{j}$. Their corresponding coefficients are $C_{u}$ and $D_{j}$. Introducing scaling and shifting parameters on above functions can be expressed as: $\phi_{j, k}(t)=2^{j / 2} \phi\left(2^{j} t-k\right)$ and $\quad \psi_{j, k}(t)=2^{j / 2} \psi\left(2^{j} t-k\right)$; where $\varphi(t)$ and $\psi(t)$ are the mother function. The difference two adjacent scaling space $V_{j}$ and $V_{j+l}$ i.e. $e_{j}$ along $W_{j}$ is expressed as weighted sum of wavelet function $\psi_{j, k}(t)$ with integral shifting of $k$ but keeping the scaling parameter $j$ fixed can be expressed as,

$e_{j}=\sum_{k} D_{j}(k) \psi_{j, k}(x)$

(2)

In one dimensional wavelet transform the expansion of any function $f(x)$ is expressed as:

$f(x)=$ weighted sum of scaling function $\varphi_{u, k}(x)$ under subspace $V_{u}+$ weighted sum of wavelet function $\psi_{u, k}(x)$ under $W_{u}+$ weighted sum of wavelet function $\psi_{u+1, k}(x)$ under $W_{u+1}+$ weighted sum of wavelet function $\psi_{u+2, k}(x)$ under $W_{u+2}+\ldots \ldots \quad \ldots$

Where for a fixed scaling parameter $u$ the shifting parameter $k$ varies from 0 to some integer such that both $\varphi_{u, k}(x)$ and $\psi_{u, k}(x)$ covers the entire range of $f(x)$. The relation between the function $f(x)$ and the two basis function can be expressed as:

$$
f(x)=\sum_{k} C_{u}(k) \varphi_{u, k}(x)+\sum_{j=u}^{\infty} \sum_{k} D_{j}(k) \psi_{j, k}(x)
$$

where the staring scale $u$ can be any value since the entire signal space can be expressed as:

$$
\begin{aligned}
& L^{2}(R)=V_{0} \oplus W_{0} \oplus W_{1} \oplus W_{2} \oplus \ldots \quad \ldots \quad \ldots \\
& =V_{u} \oplus W_{u} \oplus W_{u+1} \oplus W_{u+2} \oplus \ldots \quad \ldots \quad \ldots
\end{aligned}
$$

(4)

$$
\text { Where } \oplus_{\text {denotes the union of spaces. }}
$$

The coefficients can be evaluated like Fourier series as:

$$
\begin{aligned}
& C_{u}(k)=\left\langle f(x), \varphi_{u, k}(x)\right\rangle \text { and } \\
& D_{j}(k)=\left\langle f(x), \psi_{j, k}(x)\right\rangle
\end{aligned}
$$

(5)

In the DWT under filter bank concept, the signal is passed through LP and HP filter produce approximate and detail component. Only approximate portion is further passed through LP and HP filter and so on but detail component is kept fixed. For perfect reconstruction of signal the following conditions must hold.

$$
\begin{aligned}
& G_{0}(z)=-H_{1}(-z) \\
& G_{1}(z)=H_{0}(-z)
\end{aligned}
$$

$G_{0}(z) G_{1}(z)+H_{0}(z) H_{1}(z)=2 z^{-k} ;$ where $k$ is odd, $H_{0}(z)$ and $H_{1}(z)$ are the transfer function of LP and HP analysis filter, $G_{0}(z)$ and $G_{1}(z)$ are that of LP and HP of synthesis filter.

In WPT both the detail and approximation coefficients are decomposed to create the full binary tree. For $n$ levels of decomposition the WPD produces $2 n$ different sets of coefficients (or nodes) where DWT produce only $(3 n+1)$ coefficients. The most widely used basis algorithm is entropy (cost function) based decomposition proposed by Coifman and Wickerhauser to find the best tree. Above concept will be used in this paper to extract the features of an image.

In image processing two dimensional scaling and wavelet functions are used like:

$$
\begin{aligned}
& \phi_{u, m, n}(x, y)=2^{u / 2} \phi\left(2^{u} x-m, 2^{u} y-n\right) \\
& \psi_{u, m, n}^{s}(x, y)=2^{u / 2} \psi^{s}\left(2^{u} x-m, 2^{u} y-n\right) ; \\
& s=\{H, V, D\}
\end{aligned}
$$

Two dimensional wavelet transform measure the variation of $f(x, y)$ along $H$ (horizontal), $V$ (vertical) and $D$ (diagonal) shown by index $s$ corresponding to wavelet function but the scaling function provides only approximate component.

This section provides three examples pertinent to above concept of wavelet transform.

\subsection{Example-1}

Consider a signal, $\quad y=\left\{\begin{array}{ll}\ln (x) & 0 \leq x \leq 1 \\ 0 & \text { otherwise }\end{array}\right.$ determine the coefficients $C_{0}(0), C_{1}(0), C_{1}(1), D_{0}(0), D_{1}(0), D_{1}(1)$, $D_{2}(0), D_{2}(1), D_{2}(2)$ and $D_{2}(3)$.

The MATLAB code provides the following coefficients:

$C_{0}(0)=-0.9990, C_{1}(0)=-1.1959, C_{1}(1)=-0.2169, D_{0}(0)$ $=-0.6922, \quad D_{1}(0)=-0.4889, \quad D_{1}(1)=-0.1201, \quad D_{2}(0)=$ $0.3451, D_{2}(1)=-0.0851, D_{2}(2)=-0.0504$ and $D_{2}(3)=$ 0.0358 .

\subsection{Example-2}

Solve the fig. 7.19, page 498 of Rafael C. Gonzalez and Richard E. Wood, Digital Image Processing, Third edition, Pearson education, 2013 using Matlab.

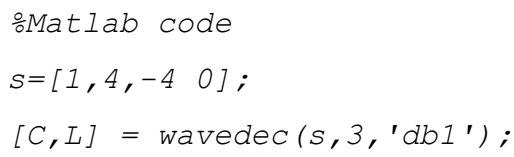

$\%$ To extract the level 3 approximation coefficients

$c A 3=\operatorname{appcoef}\left(C, L, d^{\prime} b I^{\prime}, 3\right) ;$ 
\%To extract the levels 3, 2, and 1 detail coefficients from $C$, type

$[C D 1, C D 2, C D 3]=\operatorname{detcoef}(C, L,[1,2,3])$;

Let us reconstruct the signal from approximation and the details of level 1, 2 and 3 .

$$
\begin{aligned}
& A 3=\operatorname{wrcoef}\left(' a,, C, L, ' d b 11^{\prime}, 3\right) \text {; } \\
& D 1=\operatorname{wrcoef}\left(' d, C, L,{ }^{\prime} d b 1 \text { ', } 1\right) ; \\
& D 2=\operatorname{wrcoef}\left({ }^{\prime} d ', C, L, ' d b 1\right. \text { ',2), } \\
& \text { D3 }=\operatorname{wrcoef}(' d,, C, L, ' d b 1 \text { ',3); } \\
& A 3+D 1+D 2+D 3
\end{aligned}
$$

\subsection{Example-3}

Fig.1 shows the 2 level decomposition of an image of Jahangirnagar University campus (Amar Ekushey).
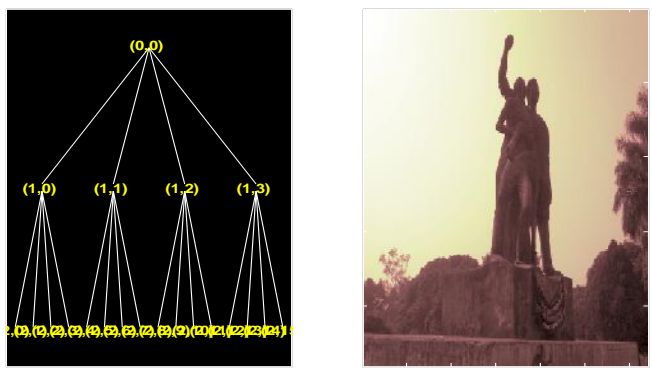

(a) Element $(1,0)$ of the wavelet packet tree
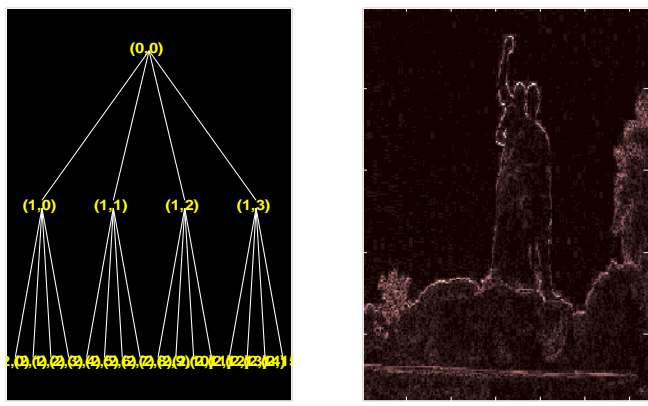

(b) Element $(1,1)$ of the wavelet packet tree
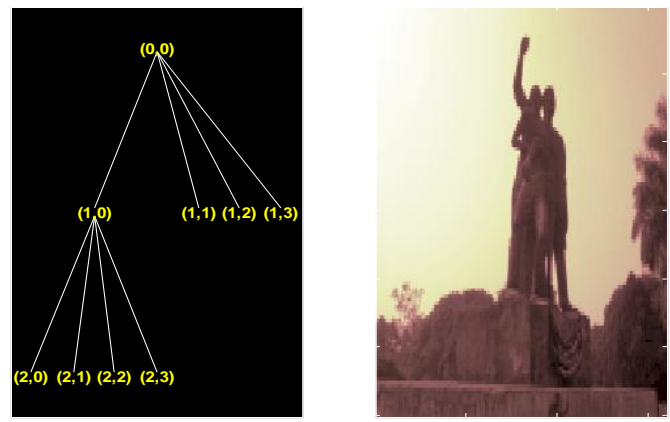

(c) Element (2,0 ) of best tree

Fig 1: Two level decomposition of an image using WPT and its best tree

\section{METHODOLOGY}

An image can be thought as the weighted sum of basis function with proper scaling and phase shift. Similar concept is also applicable in two dimensional DWT; where the basis function is a matrix. Weighted sum of only few largest coefficient can represented the image approximately which is the basic idea of image compression. The algorithm regarding compression of an image using DWT is like:

(a) Select a wavelet filter for example symlet

(b) Apply DWT till level $J$

(c) Sort the wavelet coefficient is ascending order

(d) Select top $10 \%$ coefficients

(e) Reconstruct the image using IDWT taking the coefficients of step (d)

The aim of the paper is to identify a person using image of fingerprint. Here two algorithms are proposed: applying conventional DWT and wavelet packet transform (WPT)

\subsection{Algorithm-1}

1. Read an image of fingerprint, convert it to grayscale and resize as $256 \times 256$. Apply preprocessing on it and store the image as a variable $F$.

2. Evaluate $F_{m}=F-\bar{F}$; where $\bar{F}$ is the mean value of pixels of image $F$.

3. Apply conventional DWT on $F_{m}$ like: $F_{D W T}=D W T\left(F_{m}\right)$ and determine its concentration vector $C_{D W T}$.

4. Extract top 5\% co-efficient of $F_{D W T}$ in context of energy content and select the corresponding element $C_{D W T i}$ and index $i$ from $C_{D W T}$. Finally plot $C_{D W T i}$ vs. $i$ and the curve provides the characteristics of the fingerprint.

\subsection{Algorithm -2}

1. Read an image of fingerprint and apply preprocessing on it. Store the image as a variable $F$.

2. Evaluate $F_{m}=F-\bar{F}$; where $\bar{F}$ is the mean of image $F$.

3. Apply wavelet packet transform WPT on $F_{m}$ using 'symlet' till level $k=5$ like: $F_{D W T}=W P T\left(F_{m}, k\right.$, wavelet_type $)$.

4. Choose appropriate basis function for example FBI basis matrix of $64 \times 9$ where the non-zero elements lie in last three columns. Let us represent the submatrix of last three column as FBI_basis .

5. Extract the co-efficient of $F_{D W T}$ using FBI basis matrix using the concept of best basis function like:

$F_{F B I \_C o-e f f i c i e n t}=$ extract_basis $\left(F_{W P T}, F B I_{\_}\right.$basis $)$ 
6. Determine the concentration vector $\mathrm{C}_{\mathrm{FBI} \_ \text {Co-efficient }}$ of $\mathrm{F}_{\mathrm{FBI}} \mathrm{Co}_{-}$efficient and extract the index of the top 5\% elements of the concentration vector like model-1. Finally plot $C_{F B I_{-} C o-e f f i c i e n t_{-} i}$ vs. $i$ which provide the features of the mage.

To clarify the concept of concentration vector another example is given below.

\subsection{Example-4}

Determine concentration vector of. $\mathbf{M}=\left[\begin{array}{lll}1 & 7 & 5 \\ 2 & 4 & 8 \\ 5 & 3 & 2\end{array}\right]$

The energy of the of the matrix $\mathbf{M}$,

$S=\sum_{i=0}^{2} \sum_{j=0}^{2} M^{2}(i, j)=197$

The vector $\mathbf{D}$ taking square of elements of $\mathbf{M}$ in descending order,

$\mathbf{D}=\left[8^{2} 7^{2} 5^{2} 5^{2} 5^{2} 4^{2} 3^{2} 2^{2} 2^{2}\right]$

The first element of $\mathbf{D}$ is $82 / 197=64 / 197=0.325 ; 2$ nd element of $\mathbf{D}$ is $(82+72) / 197=0.574 ; 3$ rd element of $\mathbf{D}$ is $(82+72+52) / 197=0.701 ; \quad 4$ th element of $\mathbf{D}$ is $(82+72+52+52) / 197=0.827$ etc. Putting above values in descending order provides the concentration vector of $\mathrm{M}$ like below.

$\mathbf{C}=\left[\begin{array}{lllllllll}0.325 & 0.574 & 0.701 & 0.827 & 0.909 & 0.954 & 0.975 & 0.995 & 1\end{array}\right]^{T}$

\section{RESULTS}

First of all three images under consideration is shown in fig. 2 and 3. Applying the algorithm of image compression of previous section top $20 \%, 10 \%, 5 \%$ and $1 \%$ coefficients are selected for the fingerprint of fig. 2(a)-(d). Till $5 \%$ of coefficients the image seems to be acceptable range and beyond that it starts to distort. Similar analysis is shown another two images "Amar Ekushe" and "Sangsaptak" of Jahangirnagar University campus shown in fig. 3 and 4.

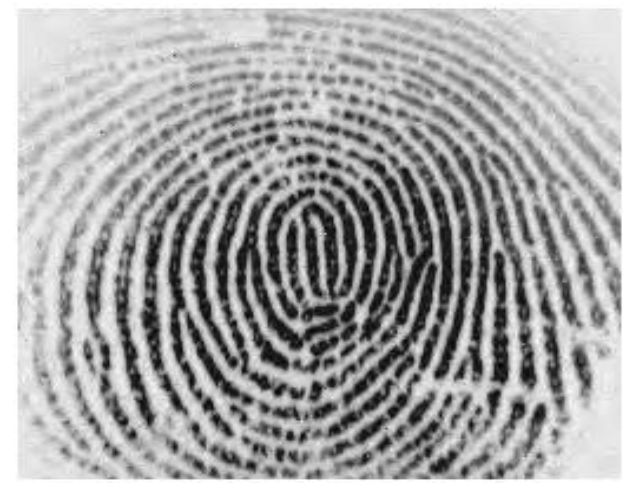

(a) Top $20 \%$ coefficients

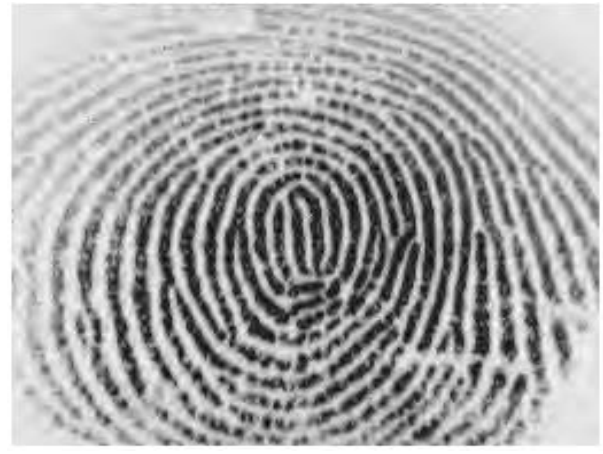

(b) Top $10 \%$ coefficients

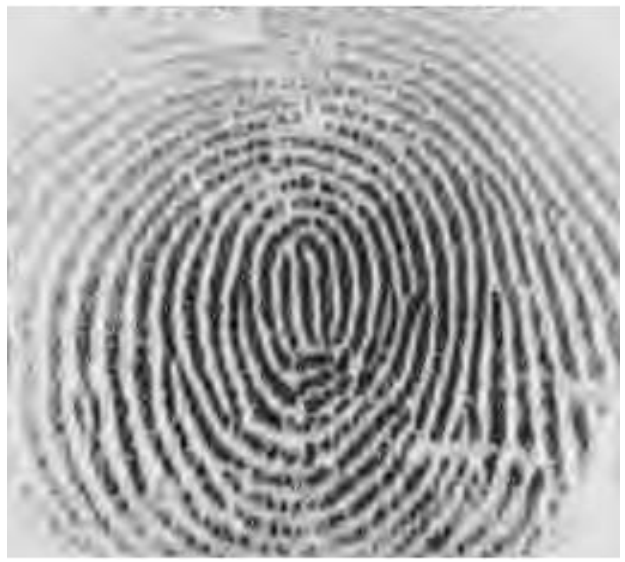

(c) Top 5\% coefficients

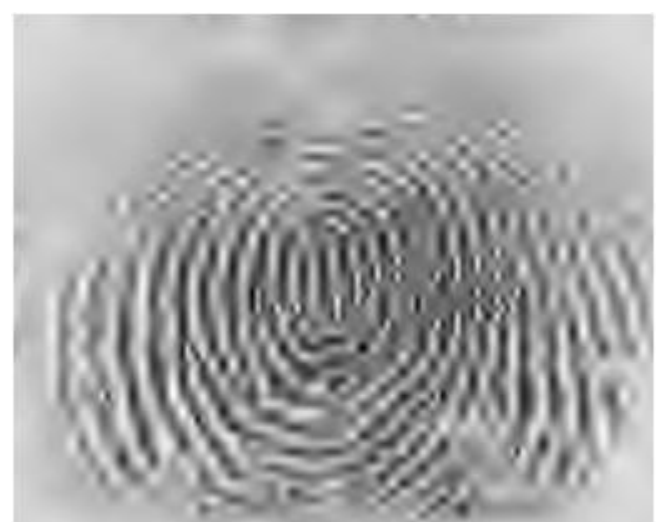

(d) Top $1 \%$ coefficients

Fig 2: Compression of image of fingerprint

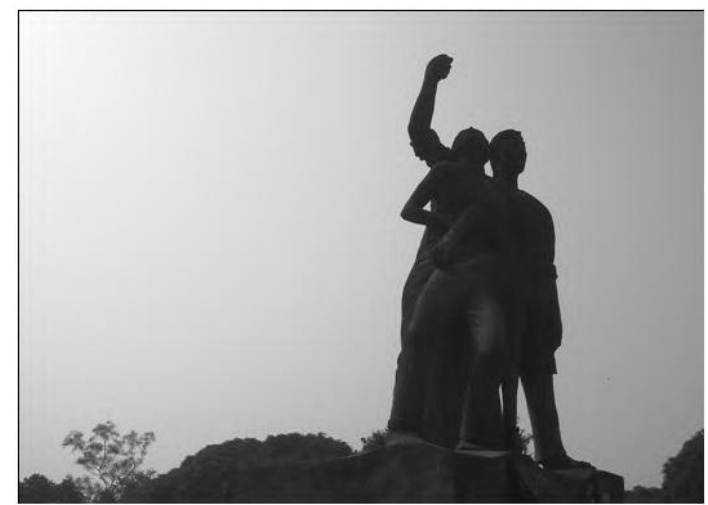

(a) Original Image Amar Ekushe 


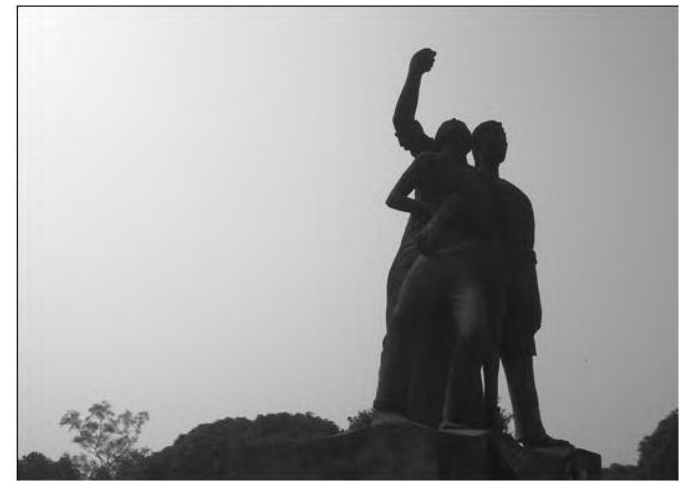

(b) $10 \%$ co-efficient

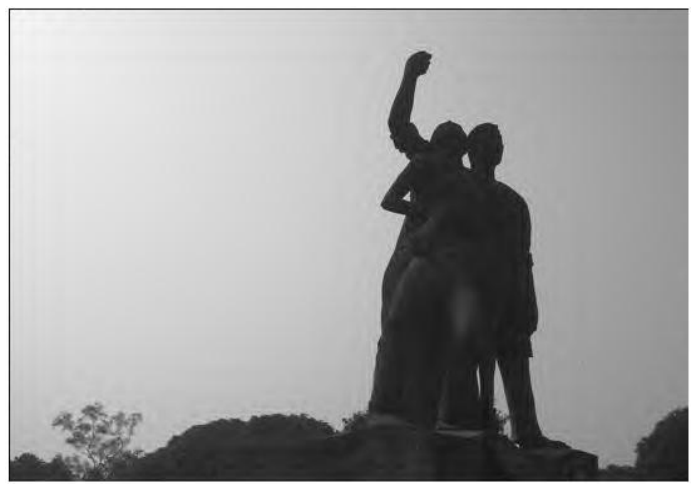

(c) $5 \%$ co-efficient

Fig 3: Compression of image of "Amar Ekushe"

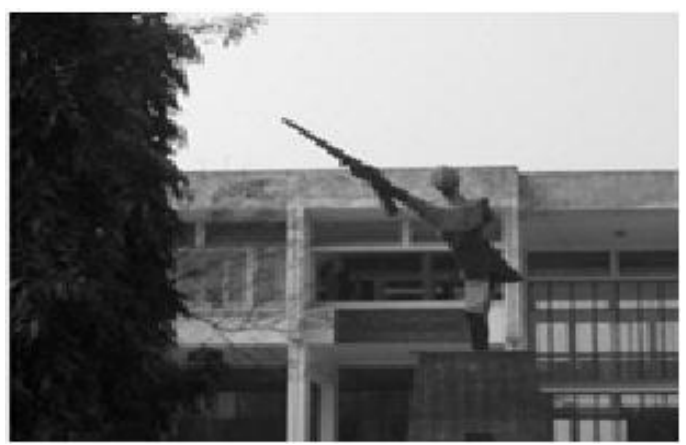

(a) Original Image "Central Library"

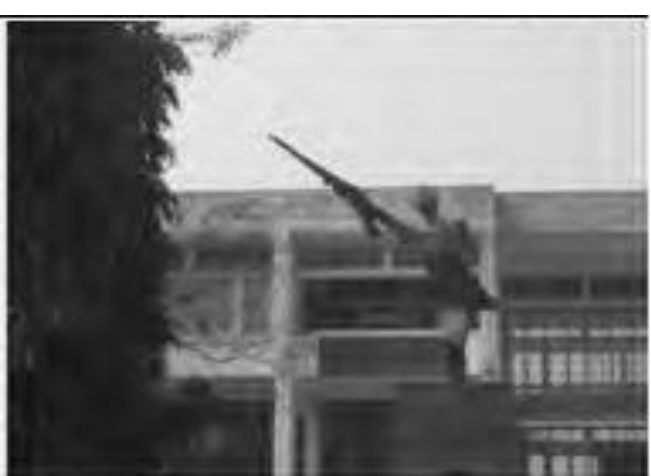

(b) $10 \%$ co-efficient

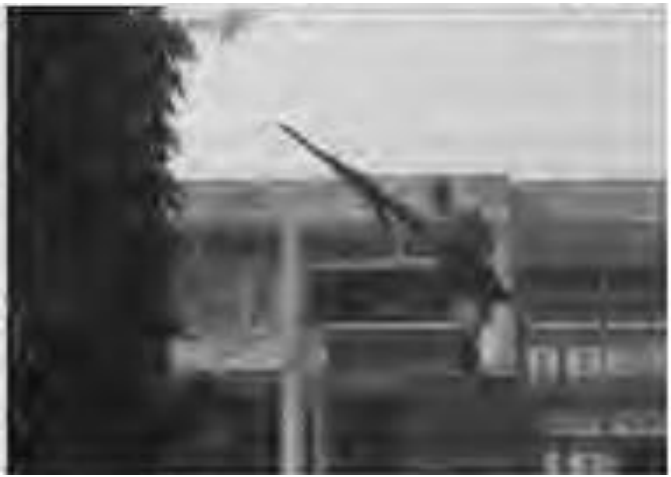

(c) $5 \%$ co-efficient

Fig 4: Compression of image of "Sangsaptak"

A sample fingerprint image is selected then its top 5\% coefficients are acquired. The every element of a matrix has a corresponding element in concentration vector visualized from example-3. The elements of concentration vector corresponding to the selected coefficients and the corresponding index is plotted in fig. 5 for both DWT and WPT.

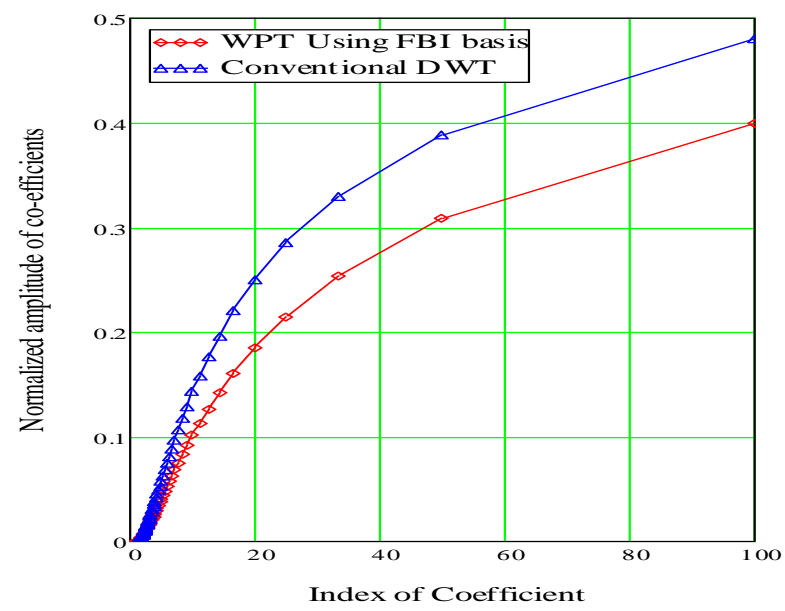

Fig 5: Amplitude of top 5\% coefficients vs. index of coefficients

Both the curves have same profile of exponential growth but the amplitude is different. The profile of normalized coefficient are again showed in fig. 6 for both conventional DWT and WPT of an image of fingerprint considering 00 and 1800 rotation. The variation of profile of normalized coefficients vs. index with 1800 rotation of the image is found very small hence system will work independent of orientation of finger on the scanner. The deviation between two curves is more prominent under WPT based on FBI basis matrix where the curves are found very closed for the case of conventional DWT. The conventional DWT is more durable on orientation of image at the expense of process time since under WPT less number of level decomposition is required using optimum basis matrix. 


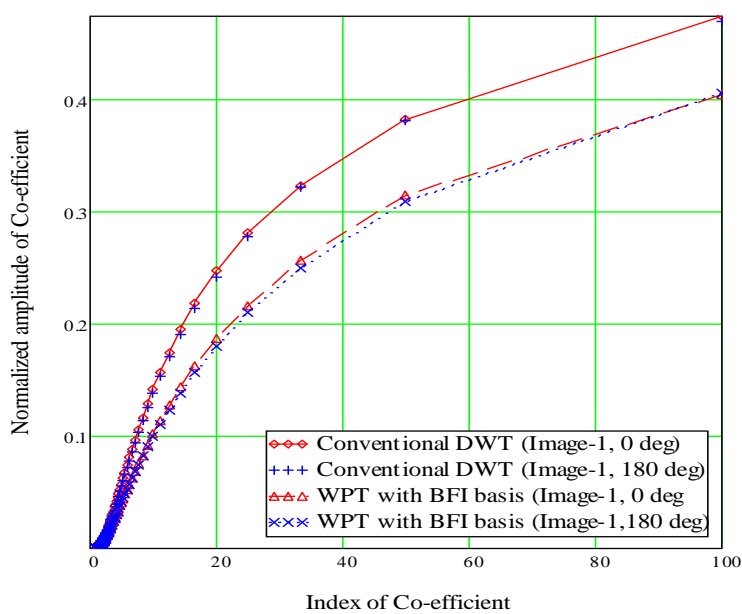

Fig 6: Profile of normalized co-efficient with 1800 rotation

Next two preprocessing operations are applied: filtering the image with a low pass Gabor filter to enhance the contrast then thinning operation is made on the enhanced image. The resultant image after above preprocessing is shown in fig. 7. The following expression of impulse response of Gabor filter is used:

$$
G(x, y)=\exp \{-V(\theta, \sigma, \gamma)\} U(\theta, \lambda, \Psi)
$$

Where $\quad U(\theta, \lambda, \Psi)=\cos \left\{2 \pi \frac{\frac{x-s}{N} \cos \theta+\frac{y-s}{N} \sin \theta}{\lambda}+\Psi\right\}$
$V(\theta, \sigma, \gamma)=\frac{\left(\frac{x-s}{N} \cos \theta+\frac{y-s}{N} \sin \theta\right)^{2}+\gamma^{2}\left(\frac{-x+s}{N} \sin \theta+\frac{y-s}{N} \cos \theta\right)^{2}}{2 \sigma^{2}}$

$\theta$ is the rotation angle of the Gaussian envelope.

$S$ is the location of the peak of the Gaussian envelope

$\sigma^{2}$ is the variance of the envelope

$\psi$ is the phase shift parameter

$\gamma$ governs the shape of footprint of the filter from elliptic to circular for a particular phase $\psi$

$N$ is the normalized parameter provides the range of $x$ and $y$ to fix the peak amplitude of the characteristics curve

$\lambda$ is another shape parameter its fractional value provides ripples or side lobe on the characteristics curve.

We took the parameters of the filter as: $S=80, N=80, \lambda=$ $1.2, \sigma=0.015, \gamma=2, \psi=0$ and $\theta=\pi / 2$. Now the spectrum of the image is different and impact of frequency content of the image will be reflected on the normalized co-efficient vs. index profile of fig. 8 .

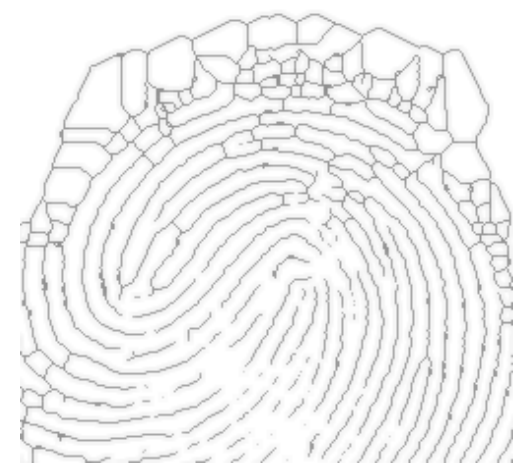

(a) Image-1

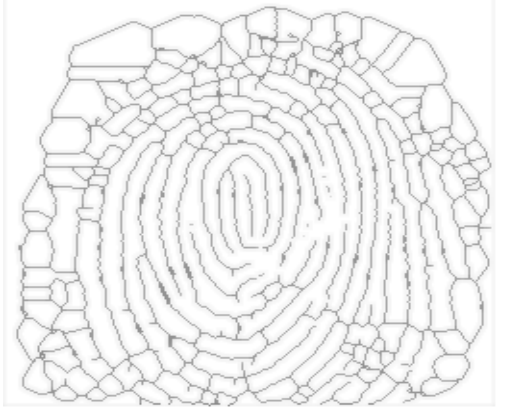

(b)Image-2

Fig 7: Fingerprints after application of thinning scheme on filtered image

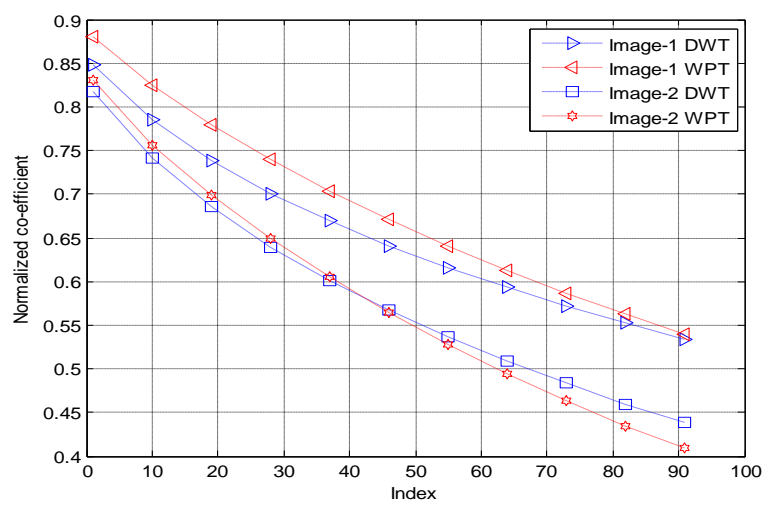

Fig 8: Comparison of images of fig.7 considering amplitude of top 5\% coefficients vs. index of coefficients

Now the difference between profiles of coefficients of DWT and WPT is wider because of truncation of medium and high frequency components of the image. We can explain the phenomenon like: the output of HP filters i.e. the detail components of the filter bank become less prominent therefore in convention DWT only the approximate component carries the features of the images of fig. 6 but in WPT both the approximate and the details components are splited hence other components also carry the features of the image hence arrangement of coefficients of the energetic component will be different visualized from fig. 8 . The accuracy of above case is found better than the previous case at the expense of process time. The benchmarked database of fingerprint is taken and only 50 of them are taken for analysis. Considering the average variation of coefficients $1 \%$ as the threshold we got $91.831 \%$ and $91.172 \%$ accuracy for DWT and WPT respectively for the previous case but the 
accuracy is found $92.783 \%$ and $91.852 \%$ with application of Gabor filter and thinning scheme. It is worth to mention that the original image can be reconstructed from top 5\% coefficient used here.

\section{CONCLUSION}

In this paper an image is converted into a vector of few points as the features of the image can be stored in a database instead of the original image at the same time the algorithm provides a very simple way of image identification. Such scheme reduces the storage required for the image at the expense of process time. The performance can be further enhanced using morphological operation on the image prior applying DWT/WPT. The model is applicable for fingerprint identification even for big objects like font detection. Still we have some pending jobs like: comparison of the proposed scheme with other models in context of accuracy rate and process time. Finally the concept of the paper is also applicable in other biometric identifications like: human face and retina detection even it is applicable in feature extraction of biomedical signals like: ECG and EEG.

\section{REFERENCES}

[1] De Stefano and P. R. White, "Training Methods for Image Noise Level Estimation onWavelet Components," EURASIP Journal on Applied Signal Processing 2004:16, 2400-2407

[2] Rafael C. Gonzalez and Richard E. Wood, Digital Image Processing, Third edition, Pearson education, 2013

[3] Jagannath Sethi1, Sibaram Mishra, Prajna Parimita Dash, Sudhansu Kumar Mishra and Sukadev Meher, "Image Compression Using Wavelet Packet Tree," ACEEE Int. J. on Signal \& Image Processing, Vol. 02, No. 01, Jan 2011, pp.41-43.

[4] G. K. Kharate and V. H. Patil, "Color Image Compression Based On Wavelet Packet Best Tree," IJCSI International Journal of Computer Science Issues, Vol. 7, Issue 2, No 3, pp.31-35, March 2010

[5] Deng Wang, Duoqian Miao and Chen Xie, "Best basis-based wavelet packet entropy feature extraction and hierarchical EEG classification for epileptic detection," Expert Systems with Applications, Elsevier Ltd, vo.38, pp.14314-14320, 2011

[6] Aguerrebere, G. Capdehourat, M. Delbracio, M. Mateu, A. Fern' andez and F. Lecumberry. "An Improved Face Recognition Algorithm through Gabor Filter Adaptation," Automatic Identification Advanced Technologies, 2007 IEEE Workshop, pp.74-79, June 2007

[7] Muhammad Sharif, Adeel Khalid, Mmudassar Raza and Sajjad Mohsin, "Face Recognition using Gabor Filters," Journal of Applied Computer Science \& Mathematics, no. 11 (5),.pp.53-58, 2011, Suceava

[8] C. Aguerrebere, G. Capdehourat, M. Delbracio, M. Mateu, A. Fern'andez and F. Lecumberry. "An Improved Face Recognition Algorithm through Gabor Filter Adaptation," Automatic Identification Advanced Technologies, 2007 IEEE Workshop, pp.74-79, June 2007
[9] Tsai, J. S. Taur and C. W. Tao, "Iris Recognition Using Gabor Filters and the Fractal Dimension," Journal of Information Science and Engineering 25, 633-648 (2009)

[10] M. Rajalakshmi and P. Subashini, "Texture Based Image Segmentation of Chili Pepper X-Ray Images Using Gabor Filter," International Journal of Advanced Studies in Computer Science \& Engineering IJASCSE, vol. 3, pp.44-51, Issue 3, 2014

[11] Wan Azizun Wan Adnan, Lim TZE Siang and Salasiah Hitam, "Fingerprint recognition in wavelet domain," Journal Teknologi, 41(D), Universiti Teknologi Malaysia, pp. 25-42, Dis. 2004.

[12] M. Tico, P. Kuosmanen and J. Saarinen, "Wavelet domain features for fingerprint recognition," IEE Electronics Letters, Vol.37, No. 1, pp.21-22, 4th January, 2001.

[13] Anil K. Jain, Yi Chen and Malter Demirkus, "Pores and Ridges: High-Resolution Fingerprint Matching Using Level 3 Features," IEEE Transactions on Pattern Analysis and Machine Intelligence, Vol. 29, No. 1, pp. 15-27, January 2007.

[14] K. Thaiyalnayaki, S. Syed Abdul Karim and P. Varsha Parmar, "Finger print recognition using Discrete Wavelet Transforn," International Journal of Computer Applications (0975-8887), Vol. 1, No. 24, pp. 96-100, 2010.

[15] Seok Won Lee and Boo Hee Nam, "Fingerprint Recognition Using Wavelet Transform and Probabilistic Neural Network," International Joint Conference on Neural Networks, 1999. IJCNN'99, Vol. 5, DOI: 10.1109/IJCNN.1999.836183, pp. 3276-3279, July 10-16, 1999, Wahsington, DC, USA.

[16] Dhruv Batra, Girish Singhal and Santanum Chaudhury, "Gabor Filter based Fingerprint Classification using Support Vector Machines," IEEE India Annual Conference 2004, pp. 256-261, DECEMBER 20-22, INDICON 2004.

[17] Chih-Jen Lee and Sheng-De Wang, "Fingerprint feature extraction using Gabor filters," Electronics Letters, vol.35, no. 4, pp.288-290, February 18, 1999.

[18] G. Sambasiva Rao, C. Naga Raju, L.S.S Reddy and E. V. Prasad, "A Novel Fingerprints Identification System Based on The Edge Detection," IJCSNS International Journal of Computer Science and Network Security, Vol. 8, No. 12, pp. 394.397, December 2008.

[19] Brian DeCann, Bozhao Tan and Stephanie Schuckers, "A Novel Region Based Liveness Detection Approach for Fingerprint Scanners," Advances Biometrics, Volume 5558, pp 627-636, 2009

[20] Josef Strom Bartunek, Mikael Nilsson, Jorgen Nordberg and Ingvar Claesson, "Neural Network based Minutiae Extraction from Skeletonized Fingerprints," IEEE Region 10 Conference 2006, TENCON 2006, pages 1-4, DOI: 10.1109/TENCON.2006.344104, November 14-17, 2006, Hong Kong. 